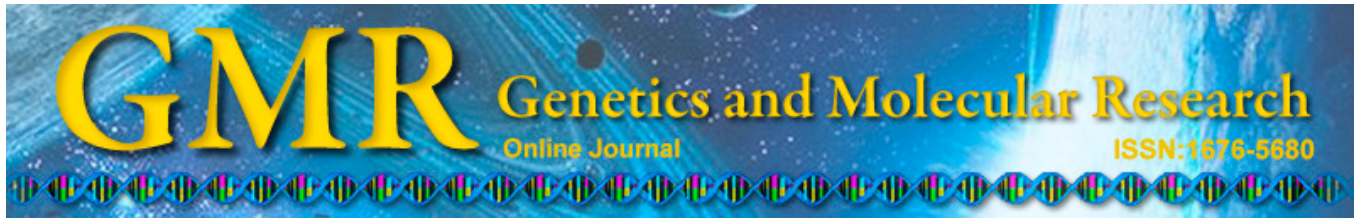

\title{
MYH9 gene polymorphisms may be associated with cerebrovascular blood flow in patients with type 2 diabetes
}

\author{
C. Ling ${ }^{1}$, C.Y. Cai ${ }^{1}$, B.C. Chang ${ }^{2}$, W.T. Shi ${ }^{1}$, F.J. Wei ${ }^{1}$, P. Yu ${ }^{1}$, L.M. Chen ${ }^{2}$ \\ and W.D. $\mathbf{L i}^{1}$ \\ ${ }^{1}$ Research Center of Basic Medical Sciences, Tianjin Medical University, \\ Tianjin, China \\ ${ }^{2}$ Metabolic Diseases Hospital, Tianjin Medical University, Tianjin, China \\ Corresponding author: W.D. Li \\ E-mail: liweidong98@tijmu.edu.cn
}

Genet. Mol. Res. 14 (1): 1008-1016 (2015)

Received January 7, 2014

Accepted March 24, 2014

Published February 6, 2015

DOI http://dx.doi.org/10.4238/2015.February.6.4

\begin{abstract}
Genetic factors play an important role in type 2 diabetes (T2D) complications. Alteration of cerebrovascular blood flow (CBF) is a direct result of cerebrovascular diseases. However, few studies have reported the role of genetics on CBF in patients with T2D. We investigated whether single-nucleotide polymorphisms (SNPs) in metabolic disease genes are associated with $\mathrm{CBF}$ in patients with T2D. CBF velocities of CBF were measured in 337 Han Chinese patients with T2D using transcranial Doppler sonography, with 54 cerebrovascular blood flow parameters documented. Fifty-two SNPs from 31 metabolic disease candidate genes were genotyped in patients. Quantitative allelic association and haplotype analyses were performed for candidate gene SNPs and CBF phenotypes. Spearman correlation was used to determine the relationship between CBF parameters and basic clinical characteristics, particularly, body mass index, lipids, fibrinogen, and GHbA1c. MYH9 gene SNPs (rs875726 and rs735853) may be associated with the peak velocity of the right-middle cerebral
\end{abstract}


artery. SNPs rs875726, rs2009930, and rs375246 of the MYH9 gene may be associated with the mean velocity of the right-anterior and posterior cerebral artery. The haplotype G-C-A (rs2239782-rs3752462rs2269532) of MYH9 may be associated with CBF. MYH9 gene polymorphisms may be associated with multiple CBF phenotypes in Chinese patients with T2D. However, whether MYH9 is a candidate gene for cerebrovascular diseases in Chinese patients with T2D remains unknown.

Key words: Association study; Cerebrovascular blood flow; GHbA1c; MYH9

\section{INTRODUCTION}

The brain is one of the most perfused organs in the body, receiving $15-20 \%$ of the total cardiac output. The high oxidative metabolic status of the brain relies on both high cardiac output and relatively constant cerebrovascular blood flow (CBF). CBF must be precise, as both hyperemia and ischemia can damage brain tissue or cause death of brain cells. The Global Burden of Disease Study predicted that cerebrovascular disease will be the second leading cause of death from 1990-2020 (American Diabetes Association, 1997). In patients with type 2 diabetes (T2D), the risk of stroke is increased 2- to 3-fold, while the risk of death is increased 2-fold compared to patients without diabetes (Almdal et al., 2004). Diabetes is an independent risk factor for death from stroke (Tuomilehto et al., 1996), and it can increase the risk of cerebrovascular disease through its effects on blood flow regulation (Novak et al., 2006) and even drive cerebrovascular remodeling (Harris et al., 2005), directly altering CBF.

Preliminary studies showed that genetic factors play an important role in cerebrovascular diseases. The $\mathrm{C}$ allele of codon 29 of the translated sequence of transforming growth factor-betal gene is a susceptibility allele for cerebral infarction in Japanese patients with T2D (Katakami et al., 2011). The $1908 \mathrm{~T}$ allele of the $L M N A$ gene is generally associated with cerebral vascular disease, but it is not related to age, hypertension, total cholesterol, or triglycerides (Liang et al., 2005). However, the global phenotype of CBF in patients with T2D has not been thoroughly examined. In particular, whether T2D-related single-nucleotide polymorphisms (SNPs) contribute equally to the alteration of CBF remains unknown. Importantly, intensive glucose control does not affect the incidence of stroke in patients with T2D (Marso et al., 2010). Thus, we investigated whether T2D candidate genes are also associated with CBF.

\section{MATERIAL AND METHODS}

\section{Patients}

A cohort of 337 hospitalized Chinese patients with T2D was recruited from the Metabolic Diseases Hospital of Tianjin Medical University. T2D was diagnosed according to criteria of the American Diabetes Association (1997). This study was conducted according to the Declaration of Helsinki and was approved by the Committee on Studies Involving Human Beings at Tianjin Medical University. All subjects provided written informed consent prior to this study. Clinical data were recorded using Filemaker Pro 11, including age, gender, body 
mass index (BMI), T2D duration, total cholesterol (TC), triglycerides (TG), high-density lipoproteins (HDL), GHbA1c, and plasma fibrinogen (FIB). Mean age of patients was $59.2 \pm 11.1$ years, BMI was $26.24 \pm 3.48 \mathrm{~kg} / \mathrm{m}^{2}$, and T2D duration was $11.0 \pm 7.24$ years. Among these patients, 201 (59.6\%) individuals had a family history of T2D (Table 1).

\section{Table 1. Clinical characteristics of 337 subjects (189 males, 148 females).}

\begin{tabular}{|c|c|c|c|c|c|}
\hline & $\mathrm{N}$ & Minimum & Maximum & Mean & SD \\
\hline Age (years) & 337 & 27.0 & 84.0 & 59.2 & 11.1 \\
\hline BMI $\left(\mathrm{kg} / \mathrm{m}^{2}\right)$ & 334 & 15.8 & 39.92 & 26.24 & 3.48 \\
\hline DM duration (years) & 336 & 0.02 & 36.00 & 11.00 & 7.24 \\
\hline $\mathrm{TG}(\mathrm{mM})$ & 319 & 0.53 & 21.29 & 2.33 & 2.45 \\
\hline $\mathrm{TC}(\mathrm{mM})$ & 320 & 2.71 & 11.92 & 5.56 & 1.34 \\
\hline HDL (mM) & 322 & 0.80 & 3.20 & 1.48 & 0.34 \\
\hline $\mathrm{LDL}(\mathrm{mM})$ & 321 & 1.12 & 7.92 & 3.43 & 1.06 \\
\hline FIB $(g / L)$ & 323 & 1.00 & 8.40 & 3.63 & 1.07 \\
\hline GHbA1c [\% (mM)] & 310 & $5.0(31)$ & $16.3(154)$ & $8.2(66)$ & $1.8(-4)$ \\
\hline $\mathrm{AI}$ & 320 & 0.90 & 6.73 & 2.84 & 0.84 \\
\hline
\end{tabular}

$\mathrm{SD}=$ standard deviation; $\mathrm{BMI}=$ body mass index; $\mathrm{DM}=$ diabetes mellitus; $\mathrm{TG}=$ triglycerides; $\mathrm{TC}=$ total cholesterol; HDL = high-density lipoproteins; LDL = low-density lipoproteins; FIB = fibrinogen; GHbA1c = glycosylated hemoglobin A1c; AI = atherogenic index $=(\mathrm{TC}-\mathrm{HDL}) / \mathrm{HDL}$.

\section{Transcranial Doppler sonography (TCD) measurements}

CBF velocities were measured using noninvasive TCD through temporal and ocular windows. We collected 54 phenotypes from the TCD tests, including peak, mean, and minimum velocities of blood flow in the binary (anterior, middle, and posterior) cerebral arteries, vertebral arteries, and basilar arteries. In addition, the pulsatility index (PI), resistance index (RI), and systolic and diastolic ratio (S:D) were also tested.

\section{Molecular genotyping}

Thirty-one genes associated with diabetes, diabetic nephropathy, obesity, insulin resistance, and hyperuricemia were selected based on previous studies (Price et al., 2008; Wang et al., 2011). Fifty-two SNPs in candidate genes with minor allele frequencies of $>0.1$ were examined. The $M Y H 9$ and $A B I 2$ gene regions were well covered $\left(\mathrm{r}^{2}>0.8\right)$. Genomic DNA was extracted from peripheral blood using the high-salt method. SNPs were genotyped using the Sequenom MassARRY iPLEX ${ }^{\mathrm{TM}}$ platform (Sequenom, San Diego, CA, USA), which is based on matrix-assisted laser desorption ionization-time of flight-mass spectrometry (MALDI-TOF MS) technology, at the Beijing Genome Institute. Hardy-Weinberg equilibrium (HWE) was tested using the PLINK (version 1.07) program (Purcell et al., 2007) (http://pngu.mgh.harvard.edu/purcell/plink/) (Table 2).

\section{Statistical analysis}

Outliers ( $\pm 4 \mathrm{SD}$ from the mean) were deleted prior to analysis. To screen for the influence of potential non-genetic factors on CBF, Spearman correlation was performed for 54 parameters of $\mathrm{CBF}$ and age, gender, T2D duration, lipid profiles [TC, TG, HDL, low-density 
lipids (LDL), very low-density lipids (VLDL)], FIB, and GHbA1c (Table S1). Linear regressions were performed for each CBF parameter against age within gender, and standardized residuals were saved such that the mean $=0$ and $\mathrm{SD}=1$ for each parameter for quantitative association studies. Associations between genotypes (52 SNPs) and the 54 quantitative CBF parameters, as well as haplotype association analyses, were performed using the PLINK software (version 1.07) (2007). General statistical analyses were performed using the SPSS software (SPSS Inc., Chicago, IL, USA).

\begin{tabular}{|c|c|c|c|c|c|c|c|c|c|}
\hline CHR & SNP & Gene & HWE-P & MAF & CHR & SNP & Gene & HWE-P & MAF \\
\hline 2 & rs16867321 & $U B E 2 E 3$ & 0.496 & 0.285 & 8 & rs13266634 & SLC $30 A 8$ & 0.653 & 0.265 \\
\hline 2 & rs11675251 & $A B I 2$ & 0.590 & 0.380 & 9 & rs10811661 & $C D K N 2 A / B$ & 0.837 & 0.211 \\
\hline 2 & rs3731652 & $A B I 2$ & 0.320 & 0.490 & 10 & rs7923837 & HHEX & 0.348 & 0.409 \\
\hline 2 & rs62183937 & $A B I 2$ & 0.346 & 0.250 & 10 & rs7903146 & $T C F 7 L 2$ & 0.111 & 0.221 \\
\hline 2 & rs1376877 & $A B I 2$ & 0.501 & 0.373 & 12 & rs7312112 & $I G F 1$ & 0.542 & 0.479 \\
\hline 2 & rs11677793 & SPAG16 & 0.477 & 0.273 & 12 & rs11067076 & $T B X 5$ & 1.000 & 0.189 \\
\hline 2 & rs7578326 & IRSI & 0.324 & 0.298 & 12 & rs11067083 & TBX5 & 0.614 & 0.193 \\
\hline 3 & rs2929402 & PCAF & 1.000 & 0.431 & 13 & rs371276 & SLITRK5 & 0.330 & 0.270 \\
\hline 3 & rs4402960 & $I G F 2 B P 2$ & 0.456 & 0.353 & 13 & rs409762 & SLITRK5 & 0.334 & 0.269 \\
\hline 4 & rs13129697 & $S L C 2 A 9$ & 0.919 & 0.422 & 14 & rs11624704 & $N R X N 3$ & 0.241 & 0.119 \\
\hline 4 & rs1014290 & $S L C 2 A 9$ & 0.596 & 0.296 & 15 & rs12102171 & SMAD3 & 0.814 & 0.263 \\
\hline 4 & rs6856526 & LPHN3 & 0.553 & 0.137 & 16 & rs17818920 & FTO & 0.374 & 0.266 \\
\hline 4 & rs2231142 & $A B C G 2$ & 0.812 & 0.137 & 20 & rs4814615 & PCSK 2 & 0.129 & 0.275 \\
\hline 6 & rs10946398 & $C D K A L 1$ & 0.612 & 0.427 & 22 & rs2106294 & LIMK2 & 0.678 & 0.156 \\
\hline 6 & rs7756992 & $C D K A L 1$ & 0.538 & 0.428 & 22 & rs5749286 & SFII & 0.294 & 0.264 \\
\hline 6 & rs881858 & VEGFA & 0.861 & 0.363 & 22 & rs5753669 & SFII & 0.086 & 0.265 \\
\hline 6 & rs9395706 & PKHDI & 0.831 & 0.268 & 22 & rs2295251 & SFII & 0.600 & 0.413 \\
\hline 6 & rs722208 & ESRI & 0.760 & 0.417 & 22 & rs875726 & МYH9 & 0.779 & 0.300 \\
\hline 7 & rs1581498 & $I L-6$ & 0.447 & 0.390 & 22 & rs2009930 & МYH9 & 0.577 & 0.300 \\
\hline 7 & rs768403 & $G B X I$ & 1.000 & 0.466 & 22 & rs2239783 & МYH9 & 0.096 & 0.390 \\
\hline 7 & rs386956 & $N U B 1$ & 0.258 & 0.400 & 22 & rs3752462 & МYH9 & 0.397 & 0.310 \\
\hline 7 & rs7805834 & NUBI & 0.742 & 0.139 & 22 & rs 2269532 & МYH9 & 0.746 & 0.230 \\
\hline 7 & rs446886 & $N U B 1$ & 0.763 & 0.321 & 22 & rs2071731 & МYH9 & 0.745 & 0.220 \\
\hline 8 & rs1526167 & TOX & 0.613 & 0.460 & 22 & rs739097 & МYH9 & 0.861 & 0.160 \\
\hline 8 & rs2726557 & TOX & 0.102 & 0.490 & 22 & rs 735853 & МYH9 & 0.603 & 0.242 \\
\hline 8 & rs11777927 & TOX & 0.819 & 0.380 & 22 & rs738409 & PNPLA3 & 0.504 & 0.246 \\
\hline
\end{tabular}

$\mathrm{CHR}=$ chromosome; $\mathrm{SNP}=$ single-nucleotide polymorphism; HWE-P $=$ P value of Hardy-Weinberg equilibrium test; $\mathrm{MAF}=$ minor allele frequency.

\section{RESULTS}

\section{High GHbA1c level is weakly correlated with CBF in patients with T2D}

Of the 337 Chinese T2D subjects, 12.5\% (42/337) had histories of cerebrovascular disease. Lipid profiles (TC, LDL, HDL, and VLDL) and FIB levels of patients did not significantly differ from the international standard healthy values. TG $(2.33 \pm 2.45 \mathrm{mM})$ was increased $(<1.7 \mathrm{mM})$, while the GHbA1c level $(8.2 \pm 1.8 \%$; $66 \pm 4 \mathrm{mM})$ on the day of hospitalization was significantly abnormal at $3.5-5.5 \%(3.0-6.2 \mathrm{mM})$. In this study, CBF was strongly correlated with age and diabetes mellitus $(\mathrm{DM})$ duration $(\mathrm{P}=0.00)$, and was moderately correlated with gender, BMI, FIB, and TG $(\mathrm{P}<0.05)$. However, few lipid profiles were correlated $(\mathrm{P}=0.05)$ with any of the $54 \mathrm{TCD}$ phenotypes. GHbA1c was correlated with only 1 $\mathrm{CBF}$ measurement [right-posterior cerebral artery (RPCA), peak velocity, $\mathrm{r}_{\mathrm{s}}=0.18, \mathrm{P}=0.00$ ] (Table S1). 


\section{Polymorphisms of $\mathrm{MYH} 9$ may be associated with $\mathrm{CBF}$}

To determine whether T2D-related genes are associated with CBF in patients with T2D, we performed quantitative association studies for candidate gene SNPs. Interestingly, we found that 8 SNPs of MYH9 were generally associated with CBF parameters, including 2 MYH9 SNPs (rs875726, rs735853) with a peak velocity of the right-middle cerebral artery (P $=0.0044$ and 0.0019$)$ and 3 SNPs (rs875726, rs2009930, and rs375246) with mean velocity of the RPCA and the right-anterior cerebral artery $(\mathrm{P}=0.003,0.0037$, and 0.0087 , respectively). Haplotype analysis of $M Y H 9$ candidate SNPs revealed that the G-C-A haplotype of rs2239782, rs3752462, and rs2269532 was generally associated with extensive TCD phenotypes of the cerebral arteries (Table 3), particularly with the minimum velocity of RPCA $(\mathrm{P}=0.0003)$.

\begin{tabular}{|c|c|c|c|c|c|c|c|}
\hline $\mathrm{CBF}$ & SNP & Gene & $\mathrm{P}$ & $\mathrm{CBF}$ & SNP & Gene & $\mathrm{P}$ \\
\hline LACA-Peak & rs1581498 & $I L-6$ & 0.046 & RACA-PI & rs3746876 & KCNJ15 & 0.004 \\
\hline LACA-Mini & rs5753669 & SFII & 0.044 & RACA-RI & rs6856526 & LPHN3 & 0.004 \\
\hline LACA-Mean & - & - & NA & RACA-S:D & rs3746876 & KCNJ15 & 0.011 \\
\hline LACA-PI & rs2106294 & $L I M K 2$ & 0.022 & RMCA-Peak & rs 735853 & МYH9 & 0.002 \\
\hline LACA-RI & rs2106294 & $L I M K 2$ & 0.007 & RMCA-Mini & - & - & NA \\
\hline LMCA-S:D & rs 13129697 & $S L C 2 A 9$ & 0.029 & RMCA-Mean & rs7903146 & $T C F 7 L 2$ & 0.021 \\
\hline LMCA-Peak & rs7903146 & $T C F 7 L 2$ & 0.029 & RMCA-PI & rs 13266634 & SLC $30 A 8$ & 0.021 \\
\hline LMCA-Mini & rs3746876 & KCNJ15 & 0.031 & RMCA-RI & - & - & NA \\
\hline LMCA-Mean & rs446886 & $N U B 1$ & 0.010 & RMCA-S:D & - & - & NA \\
\hline LMCA-PI & rs 738409 & PNPLA3 & 0.002 & RPCA-Peak & rs 735853 & МYH9 & 0.007 \\
\hline LMCA-RI & rs 738409 & PNPLA3 & 0.017 & RPCA-Mini & rs875726 & МYH9 & 0.039 \\
\hline LMCA-S:D & rs 738409 & PNPLA3 & 0.013 & RPCA-Mean & rs 875726 & МYH9 & 0.011 \\
\hline LPCA-Peak & rs2239782 & МYH & 0.016 & RPCA-PI & rs 738409 & $P N P L A 3$ & 0.006 \\
\hline LPCA-Mini & rs7903146 & $T C F 7 L 2$ & 0.017 & RPCA-RI & rs 738409 & PNPLA3 & 0.022 \\
\hline LPCA-Mean & rs446886 & $N U B 1$ & 0.032 & RPCA-S:D & rs 738409 & $P N P L A 3$ & 0.008 \\
\hline LPCA-PI & rs446886 & $N U B 1$ & 0.015 & RV-Peak & rs875726 & МYН9 & 0.002 \\
\hline LPCA-RI & rs2269532 & МYН9 & 0.021 & RV-Mini & rs 722208 & ESRI & 0.029 \\
\hline LPCA-S:D & rs 11777927 & $T O X$ & 0.012 & RV-Mean & rs 875726 & МYH9 & 0.010 \\
\hline LV-Peak & rs 11675251 & $A B I 2$ & 0.006 & RV-PI & rs 768403 & $G B X 1$ & 0.019 \\
\hline LV-Mini & rs1581498 & $I L-6$ & 0.050 & RV-RI & rs 768403 & $G B X 1$ & 0.025 \\
\hline LV-Mean & rs1581498 & $I L-6$ & 0.022 & RV-S:D & rs16867321 & $U B E 2 E 3$ & 0.011 \\
\hline LV-PI & rs2295251 & SFII & 0.020 & BA-Peak & rs2295251 & SFII & 0.005 \\
\hline LV-RI & rs 11675251 & $A B I 2$ & 0.003 & BA-Mini & rs1581498 & $I L-6$ & 0.004 \\
\hline LV-S:D & rs 17782313 & $M C 4 R$ & 0.012 & BA-Mean & rs2295251 & SFII & 0.006 \\
\hline RACA-Peak & rs875726 & МYH9 & 0.007 & BA-PI & rs62183937 & $A B I 2$ & 0.025 \\
\hline RACA-Mini & rs875726 & МYH9 & 0.002 & BA-RI & rs62183937 & $A B I 2$ & 0.018 \\
\hline RACA-Mean & rs875726 & МYН9 & 0.003 & BA-S:D & rs3746876 & KCNJ15 & 0.044 \\
\hline
\end{tabular}

$\mathrm{CBF}=$ cerebrovascular blood flow; $\mathrm{SNP}=$ single-nucleotide polymorphism; LACA = left-anterior cerebral artery; LMCA $=$ left-middle cerebral artery; LPCA = left-posterior cerebral artery; RACA = right-anterior cerebral artery; $\mathrm{RMCA}=$ rightmiddle cerebral artery; RPCA = right-posterior cerebral artery; LV = left vertebral artery; RV = right vertebral artery; BA = basilar artery; Peak = peak flow velocity; PI = pulsatility index; RI = resistance index; $\mathrm{S}: \mathrm{D}=$ systolic and diastolic ratio.

\section{Susceptibility genes of T2D were not equally associated with CBF}

Our results showed that several previously reported T2D-related gene SNPs, including rs7578326 near the IRS1 gene (Rung et al., 2009), rs10811661 of CDKN2A/B, rs10946398 and rs 775699 of $C D K A L 1$, and rs4402960 of IGF2BP2 (Duesing et al., 2008; Omori et al., 2008), were not associated with CBF in our subjects. Moreover, rs17818920 of the FTO gene, which is associated with extreme obesity, and rs371276 and rs409762 of the neurite-modulation gene SLITRK5 showed no association with the CBF phenotypes. 


\section{DISCUSSION}

Cerebrovascular disease is one of the main complications of T2DM. Typically, CBF is determined by a number of factors such as blood viscosity, blood vessel dilation, and cerebral perfusion pressure. However, studies have begun to focus on factors contributing to $\mathrm{CBF}$ maintenance or regulation under high glucose levels. Diabetes is a genetic predisposition disease, and the genetic background of CBF in patients with diabetes has not been well established. This is the first genetic investigation of CBF in patients with T2D.

In this study, TCD was selected as a noninvasive method for mass screening of CBF. Adjusted parameter values representing the global CBF conditions of patients with T2D were used for correlation and quantitative allelic association studies. We confirmed that CBF was correlated to DM duration, age, and BMI (Table S1). However, CBF was not globally correlated with the high level of GHbA1c. GHbA1c is used to measure the amount of glucose bound to red blood cells, which indicates a patient's blood glucose control over the past 3 months. Interestingly, a recent study indicated that the relationship between $\mathrm{GHbA} \mathrm{c}$ and macrovascular complications was not as significant as that of $\mathrm{GHbA} 1 \mathrm{c}$ with microvascular complications and demonstrated no correlation with stroke (Penno et al., 2013). However, studies suggest that a higher GHbA1c level may be a serological marker to evaluate the severity and prognosis of acute brainstem infarctions ( $\mathrm{Li}$ et al., 2012). In addition, an elevated GHbA1c level (>7.5\%) was associated with an increased risk of all-cause mortality and a lower revascularization rate in elderly patients with new-onset diabetes (Twito et al., 2013). In our study, the mean GHbA1c level was $8.2 \%(10.5 \mathrm{mM})$. Although we did not identify a globally significant correlation between GHbA1c and CBF, we cannot rule out the influence of elevated GHbA1c on cerebral vascular system. The cerebral microvascular complications of T2D should be further examined to illustrate the potential effect of GHbA1c on cerebral vascular.

In this study, quantitative allelic association and haplotype analysis revealed that SNPs of the MYH9 gene may be associated with CBF in patients with T2D. Previous studies indicated that mutations in the $\mathrm{MYH9}$ gene were responsible for various platelet disorders including hereditary macrothrombocytopenia (Dong et al., 2005), May-Hegglin syndrome, Fechtner syndrome, Sebastian syndrome, Epstein syndrome (Heath et al., 2001), Alport syndrome, and progressive sensorineural deafness (Brodie et al., 1992). Genome-wide admixture scanning revealed a highly significant association between $M Y H 9$ polymorphisms and non-diabetic end-stage renal diseases in African Americans (Kao et al., 2008). In addition, a recent study suggested that SNP rs375246 is an independent predictor of reduced glomerular filtration rate in the Spanish RENASTUR cohort population (Tavira et al., 2013). In this study, 8 SNPs of the MYH9 gene were strongly associated with CBF phenotypes, and the MYH9 G-C-A haplotype of SNPs rs2239782-rs3752462-rs2269532 were associated with most of the CBF traits, particularly the minimum blood flow velocity of RPCA (Table 4). Some of these syndromes may overlap. For example, molecular genetic analysis showed that structural changes in the myosin gene had large effects on platelets, leukocytes, and megakaryocytes. The MYH9 gene polymorphisms may have some effects on the composition of blood and partly alter the blood flow velocities in patients with T2D. However, additional studies are needed to determine whether and how these SNPs influence gene expression and protein structure.

Although none of the results revealed a genome-wide association $\left(\mathrm{P}<1 \times 10^{-7}\right)$ or significant association after Bonferroni correction, it was unusual to observe multiple $M Y H 9$ associations with independent CBF phenotypes. $M Y H 9$ polymorphisms were associated with 
nearly all of the $54 \mathrm{CBF}$ phenotypes, including the 5 most significant associations in 9 cerebral arteries (Table 4). Rather than increasing sample size, detailed phenotyping can also provide self-replication for association studies.

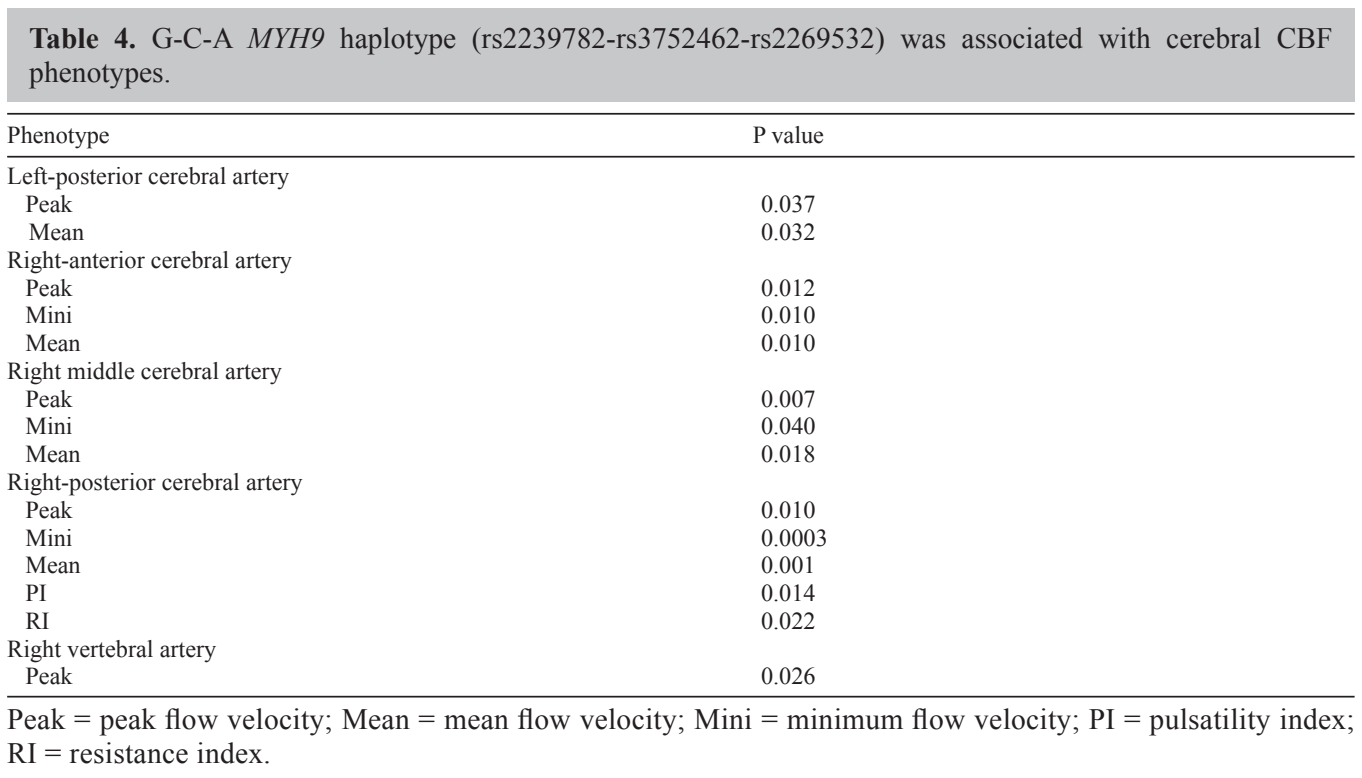

In addition to the $M Y H 9$ association, various SNPs were likely associated with middle cerebral artery (MCA) blood flow velocities. Because MCA is the most common site of cerebrovascular diseases, stenosis of the MCA increased the risk of vascular disease mortality in patients with T2D (Thomas et al., 2008). In our study, rs7903146 of TCF7L2 (Palmer et al., 2011) and rs735853 of MYH9 (Freedman et al., 2009; Cooke et al., 2012) were likely associated with right middle cerebral artery (RMCA) peak blood flow velocity $(\mathrm{P}=0.0085$, and $\mathrm{P}=0.0019$, respectively); rs12102171 of SMAD3, rs3746876 of KCNJ15 (Okamoto et al., 2010), rs5753669 of SFII (McDonough et al., 2011), and rs13266634 of SLC30A8 (Hu et al., $2009)$ with RMCA S:D (P = 0.018, 0.011, 0.014, and 0.014, respectively); SNP rs446886 of $N U B 1$ with the left middle cerebral artery (LMCA) mean blood flow velocity $(\mathrm{P}=0.01)$; SNP rs738409 of PNPLA3 with LMCA PI (P = 0.0019); and SNP rs13129697 of SLC2A9 with LMCA S:D $(\mathrm{P}=0.029)$. Many of these associations were moderate; however, several candidate gene SNPs were associated with multiple TCD phenotypes, suggesting weak but detectable contributions to the genetic relative risks of cerebrovascular diseases. In addition, SNP rs1581498 near the gene for interleukin-6 may be associated with a large spectrum of TCD phenotypes. In this study, the SNP rs 1014290 of the SLC2A9 gene was not associated with any TCD phenotype, although an association with T2D in the Han Chinese has been reported (Liu et al., 2011) (Table 3).

The genetic background of CBF is clearly polygenic and was not treated as a binary disease in our study. Because changes in blood vessel structure, hemodynamics, and blood pressure are important for $\mathrm{CBF}$, each of these factors may contribute to the overall genetic 
background. This study revealed some of these contributions and illustrated the association between metabolic disease candidate genes and CBF in patients with T2D.

In conclusion, we performed association and haplotype analyses for 52 SNPs from 31 candidate genes of metabolic diseases and found that SNPs in the MYH9 gene may be associated with CBF in Chinese patients with T2D. However, these findings require further assessment and validation within a large cohort.

\section{ACKNOWLEDGMENTS}

We wish to thank all of the patients for their cooperation. Additionally, we would like to thank the employees of the Juanjuan Wen and Jun Guo at Metabolic Diseases Hospital, Tianjin Medical University. Research supported by the Chinese National Natural Science Foundation of China (grant \#81070576) to W.D. Li.

\section{Supplementary material}

\section{REFERENCES}

American Diabetes Association (1997). Report of the Expert Committee on the Diagnosis and Classification of Diabetes Mellitus. Diabetes Care 20: 1183-1197.

Almdal T, Scharling H, Jensen JS and Vestergaard H (2004). The independent effect of type 2 diabetes mellitus on ischemic heart disease, stroke, and death: a population-based study of 13,000 men and women with 20 years of follow-up. Arch. Intern. Med. 164: 1422-1426.

Brodie HA, Chole RA, Griffin GC and White JG (1992). Macrothrombocytopenia and progressive deafness: a new genetic syndrome. Am. J. Otol. 13: 507-511.

Cooke JN, Bostrom MA, Hicks PJ, Ng MC, et al. (2012). Polymorphisms in MYH9 are associated with diabetic nephropathy in European Americans. Nephrol. Dial. Transplant. 27: 1505-1511.

Dong F, Li S, Pujol-Moix N, Luban NL, et al. (2005). Genotype-phenotype correlation in MYH9-related thrombocytopenia. Br. J. Haematol. 130: 620-627.

Duesing K, Fatemifar G, Charpentier G, Marre M, et al. (2008). Evaluation of the association of IGF2BP2 variants with type 2 diabetes in French Caucasians. Diabetes 57: 1992-1996.

Freedman BI, Hicks PJ, Bostrom MA, Comeau ME, et al. (2009). Non-muscle myosin heavy chain 9 gene MYH9 associations in African Americans with clinically diagnosed type 2 diabetes mellitus-associated ESRD. Nephrol. Dial. Transplant. 24: 3366-3371.

Harris AK, Hutchinson JR, Sachidanandam K, Johnson MH, et al. (2005). Type 2 diabetes causes remodeling of cerebrovasculature via differential regulation of matrix metalloproteinases and collagen synthesis: role of endothelin-1. Diabetes 54: 2638-2644.

Heath KE, Campos-Barros A, Toren A, Rozenfeld-Granot G, et al. (2001). Nonmuscle myosin heavy chain IIA mutations define a spectrum of autosomal dominant macrothrombocytopenias: May-Hegglin anomaly and Fechtner, Sebastian, Epstein, and Alport-like syndromes. Am. J. Hum. Genet. 69: 1033-1045.

Hu C, Zhang R, Wang C, Wang J, et al. (2009). PPARG, KCNJ11, CDKAL1, CDKN2A-CDKN2B, IDE-KIF11-HHEX, IGF2BP2 and SLC30A8 are associated with type 2 diabetes in a Chinese population. PLoS One 4: e7643.

Kao WH, Klag MJ, Meoni LA, Reich D, et al. (2008). MYH9 is associated with nondiabetic end-stage renal disease in African Americans. Nat. Genet. 40: 1185-1192.

Katakami N, Kaneto H, Osonoi T, Kawai K, et al. (2011). Transforming growth factor $\beta 1$ T868C gene polymorphism is associated with cerebral infarction in Japanese patients with type 2 diabetes. Diabetes Res. Clin. Pract. 94: e57-e60.

Li H, Kang Z, Qiu W, Hu B, et al. (2012). Hemoglobin A1C is independently associated with severity and prognosis of brainstem infarctions. J. Neurol. Sci. 317: 87-91.

Liang H, Murase Y, Katuta Y, Asano A, et al. (2005). Association of LMNA 1908C/T polymorphism with cerebral vascular disease and diabetic nephropathy in Japanese men with type 2 diabetes. Clin. Endocrinol. 63: 317-322.

Liu WC, Hung CC, Chen SC, Lin MY, et al. (2011). The rs 1014290 polymorphism of the SLC2A9 gene is associated with type 2 diabetes mellitus in Han Chinese. Exp. Diabetes Res. 2011: 527520. 
Marso SP, Kennedy KF, House JA and McGuire DK (2010). The effect of intensive glucose control on all-cause and cardiovascular mortality, myocardial infarction and stroke in persons with type 2 diabetes mellitus: a systematic review and meta-analysis. Diab. Vasc. Dis. Res. 7: 119-130.

McDonough CW, Palmer ND, Hicks PJ, Roh BH, et al. (2011). A genome-wide association study for diabetic nephropathy genes in African Americans. Kidney Int. 79: 563-572.

Novak V, Last D, Alsop DC, Abduljalil AM, et al. (2006). Cerebral blood flow velocity and periventricular white matter hyperintensities in type 2 diabetes. Diabetes Care 29: 1529-1534.

Okamoto K, Iwasaki N, Nishimura C, Doi K, et al. (2010). Identification of KCNJ15 as a susceptibility gene in Asian patients with type 2 diabetes mellitus. Am. J. Hum. Genet. 86: 54-64.

Omori S, Tanaka Y, Takahashi A, Hirose H, et al. (2008). Association of CDKAL1, IGF2BP2, CDKN2A/B, HHEX, SLC30A8, and KCNJ11 with susceptibility to type 2 diabetes in a Japanese population. Diabetes 57: 791-795.

Palmer ND, Hester JM, An SS, Adeyemo A, et al. (2011). Resequencing and analysis of variation in the TCF7L2 gene in African Americans suggests that SNP rs7903146 is the causal diabetes susceptibility variant. Diabetes 60: 662-668.

Penno G, Solini A, Zoppini G, Orsi E, et al. (2013). Hemoglobin A1c variability as an independent correlate of cardiovascular disease in patients with type 2 diabetes a cross-sectional analysis of the renal insufficiency and cardiovascular events (RIACE) Italian multicenter study. Cardiovasc. Diabetol. 12: 98.

Price RA, Li WD and Zhao H (2008). FTO gene SNPs associated with extreme obesity in cases, controls and extremely discordant sister pairs. BMC Med. Genet. 9: 4.

Purcell S, Neale B, Todd-Brown K, Thomas L, et al. (2007). PLINK: a tool set for whole-genome association and population-based linkage analyses. Am. J. Hum. Genet. 81: 559-575.

Rung J, Cauchi S, Albrechtsen A, Shen L, et al. (2009). Genetic variant near IRS1 is associated with type 2 diabetes, insulin resistance and hyperinsulinemia. Nat. Genet. 41: 1110-1115.

Tavira B, Coto E, Gómez J, Tranche S, et al. (2013). Association between a MYH9 polymorphism (rs3752462) and renal function in the Spanish RENASTUR cohort. Gene 520: 73-76.

Thomas GN, Chen XY, Lin JW, Tomlinson B, et al. (2008). Middle cerebral artery stenosis increased the risk of vascular disease mortality among type 2 diabetic patients. Cerebrovasc. Dis. 25: 261-267.

Tuomilehto J, Rastenyte D, Jousilahti P, Sarti C, et al. (1996). Diabetes mellitus as a risk factor for death from stroke. Prospective study of the middle-aged Finnish population. Stroke 27: 210-215.

Twito O, Ahron E, Jaffe A, Afek S, et al. (2013). New-onset diabetes mellitus in elderly subjects: association between HbA1c levels, mortality, and coronary revascularization. Diabetes Care 36: 3425-3429.

Wang K, Li WD, Zhang CK, Wang Z, et al. (2011). A genome-wide association study on obesity and obesity-related traits. PLoS One 6: e18939. 